\title{
An Efficient Algorithm for the Segmentation of Astronomical Images
}

\author{
Gintu Xavier, Tintu Erlin Philip, Deepthi T.V.N, K.P Soman \\ (Centre for Excellence in Computational Engg. and Networking, Amrita Vishwa Vidyapeetham, India)
}

\begin{abstract}
In this paper, an efficient algorithm for segmenting celestial objects from astronomical images is proposed. The proper segmentation of astronomical objects like planets, comets, galaxies, asteroids etc. is a difficult task due to the presence of innumerous bright point sources in the frame, presence of noise, weak edges of celestial objects, low contrast etc. In order to overcome these bottlenecks, multiple preprocessing steps are performed on the actual image prior to segmenting the desired object(s). Level Set segmentation is the key technique of this proposed method. The result of the proposed algorithm on various celestial objects substantiates the effectiveness of the proposed method.
\end{abstract}

Keywords - Classification, Level Set Segmentation, Pattern Recognition, TV Denoising, Wavelet Transform.

\section{Introduction}

Survey and statistical analysis of the ever expanding universe has been of growing concern over the past few decades. Many automated techniques have been proposed based on which the Data Mining software are built [1- 5]. Continuous evolution of the techniques is taking place to improve the performance of such a system. For most of the imaging surveys, detection and extraction of astronomical objects/sources for further classification is the primary step towards database creation $[1,3]$. The performance of a Data Mining process depends on the efficiency of all the sequential steps involved, of which segmentation is the primary step.

Efficiency of the image segmentation technique for extracting celestial object(s) in-turn affects all the bottom-up processes. Segmentation of celestial objects from the images obtained from satellite/telescope is a difficult task, due to the presence of bright point sources, noises, low contrast due to long distances and disturbances, lack of clear-cut boundaries etc. [1, 2, 4]. Previous methods employed for segmenting the astronomical objects includes watershed segmentation [6-7], binary thresholding etc. The spatial and the texture information of the astronomical objects are not retained in the above mentioned processes. The further classification of the astronomical objects becomes efficient if the texture and the shape of the objects can be retained to a great extent. The proposed algorithm takes care of these features satisfactorily. The preprocessing steps take care of the difficulties in achieving proper segmentation. The presence of point sources can adversely affect the evolution of the level set contour which is taken care by eroding the bright sources. Total Variation Denoising [13,14] applied to the Stationary Wavelet Decomposed image [11, 12] satisfactorily removes noise with edge preservation. Adaptive histogram equalization technique is used to enhance the contrast of the image. The lack of clear-cut boundary/edges for the celestial objects usually affects the segmentation. An active contour based level set segmentation technique using Signed Pressure Force (SPF) as the stopping function [22] takes care of the problem. It also offers global segmentation using a single initial contour.

The paper is structured as follows: Section II describes removal of bright point sources. Section III presents Stationary Wavelet Transform followed by TV Denoising and contrast stretching in Section IV and Section V respectively. Section VI describes the Active contour level set segmentation technique. Section VII gives the proposed algorithm and Section VIII describes the implementation steps. Results are depicted in Section IX and the conclusion is given in Section X.

\section{Removal of Bright Point Sources}

Astronomical images usually contain numerous bright point sources (stars, distant galaxies etc.). These point sources are to be primarily removed for the proper segmentation in the final stage. The removal of such abrupt change in intensities prevents the evolving level set contour, from getting stuck at the local regions. Local peak search using a matched filter or a cleaning process [3] are usually used to remove the unresolved point sources. But, multiple pass through the filter which is required during these processes, would diffuse the image and may result in the break-up of the components of the extended sources [1,3]. Erosion-a morphological 
enhancement process [8], would serve the purpose without affecting the shape and structure of the celestial objects to an extent. Matlab command 'imerode', with suitable shape and size for the structuring element removes the peak intensities satisfactorily without severe damage to the edge information.

\section{Stationary Wavelet Transform}

Wavelet Transform which is a powerful tool in image processing, is used in this method prior to image denoising. Universe is assumed to be isotropic and so are the celestial objects. Wavelet Transform does not privilege any particular direction in the image $[4,10]$ and hence is well suited for astronomical image processing.

Normal denoising causes noticeable loss of the high frequency contents, along with the smoothing of the edge features. Instead, denoising applied on wavelet transform takes care of preserving the high frequency edge components to an extent, ultimately facilitating proper segmentation. Also, it is desirable to preserve the spatial information at each level and hence Stationary Wavelet Transform rather than the translation-variant Discrete Wavelet Transform (DWT) is used. A simplest way to obtain this translational invariance is not to perform any sub-sampling [9-11]. The filters are to be up-sampled at each level of decomposition by padding low and high pass filters with zeros [10-12]. This method is commonly referred to as the "Ã Trous" algorithm $[11,12]$.

Mathematically, the SWT decomposes the image $I(x, y)$ into $J$ scales of wavelet planes $W_{j}(x, y)$ associated with the wavelet function and a smoothened plane $C_{J}(x, y)$ associated with the scaling function. This can be mathematically represented as:

$$
I(x, y)=C_{J}(x, y)+\sum_{j=1}^{J} W_{j}(x, y)
$$

The iterative process to calculate $C_{J}(x, y)$ and $W_{j}(x, y)$ can be represented as:

$$
\begin{array}{ll}
C_{0}(x, y)=I(x, y) & , \text { on initializing } J=0 \\
C_{j}(x, y)=\left\langle H_{j}, C_{j-1}\right\rangle(x, y) & , \text { for } j=1,2 \ldots \ldots J
\end{array}
$$

where,

$$
\begin{gathered}
\left\langle H_{j}, C_{j-1}\right\rangle(x, y)=\sum_{n, m} h(n, m) C_{j-1}\left(x+2^{j-1} n, y+2^{j-1} m\right) \\
W_{j}(x, y)=C_{J-1}(x, y)-C_{J}(x, y)
\end{gathered}
$$

The $W_{j}$ 's and $C_{J}$ represents the wavelet transform planes and the result of a single level SWT transform with $J=1$, is given as an example in Figure 1.

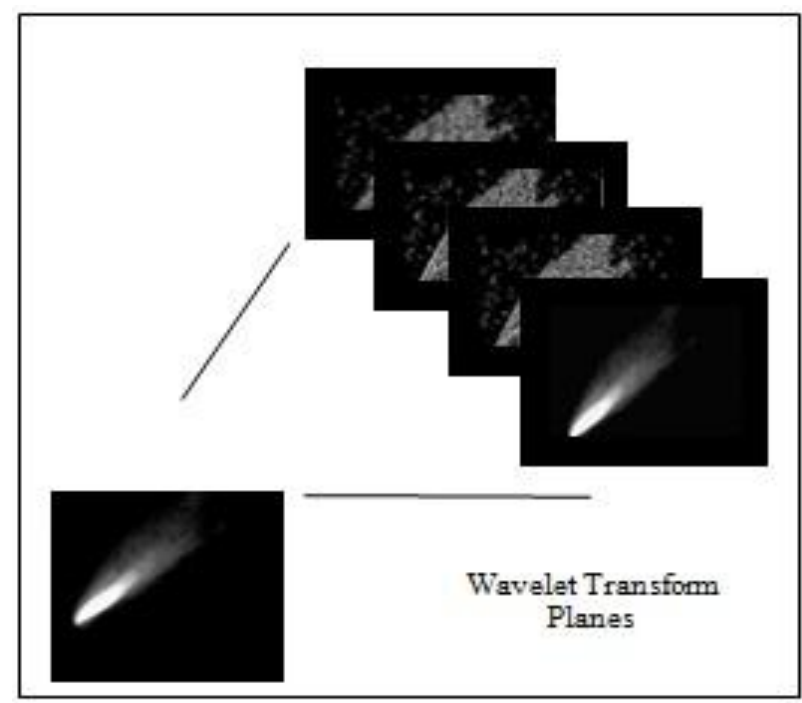

Figure 1: 1-level wavelet transform planes 


\section{TV Denoising}

Astronomical images are inherent to noise and hence need filtering to properly segment the celestial objects. Out of many denoising techniques, Total Variation denoising gives satisfactory Peak Signal-to-NoiseRatio (PSNR) value compared to other methods like Split Bregman, Edge Enhanced diffusion etc. Total Variation denoising formulation and its solution $[13,14]$ is given below.

If $U_{0}$ represents the noisy image, $U$ the clean image and $\lambda$ the control parameter, then the optimization problem is defined as:

$$
\begin{gathered}
\min _{U} E(U)=\int|\nabla U|+\lambda \int\left(U-U_{0}\right)^{2} \\
F\left(U, U_{x}, U_{y}\right)=\sqrt{U_{x}^{2}+U_{y}^{2}}+\lambda\left(U-U_{0}\right)^{2}, \text { is the objective function. }
\end{gathered}
$$

The final Euler Lagrange equation [13] for the above function is:

$$
2 \lambda\left(U-U_{0}\right)-\frac{U_{x x} U_{y}^{2}-2 U_{x} U_{y} U_{x y}+U_{y y} U_{x}^{2}}{\left(U_{x}^{2}+U_{y}^{2}\right)^{3 / 2}}=0
$$

The solution for (6) is found using gradient-descent method as:

$$
\frac{\partial U}{\partial t}=-\frac{\partial E}{\partial U}=\frac{U_{x x} U_{y}^{2}-2 U_{x} U_{y} U_{x y}+U_{y y} U_{x}^{2}}{\left(U_{x}^{2}+U_{y}^{2}\right)^{3 / 2}}-2 \lambda\left(U-U_{0}\right)
$$

This formulation is iteratively applied to the approximation and detail coefficients of the 1-level stationary wavelet transformed planes. The denoised image is retrieved using Inverse SWT (ISWT).

\section{Adaptive Histogram Equalization}

The celestial images are usually of low contrast and hence, contrast stretching would be a suitable preprocessing step before the final segmentation. The denoising performed on the image results in smoothing of the edges, making it difficult for the segmentation constraint to lock the evolving contour to the edges. Contrast stretching offers an improvement to the final segmentation process by providing enhancement to the details in the image. Here we use Adaptive Histogram Equalization (AHE) which differs from ordinary histogram equalization $[6,15,16]$. This method brings out more details in the image compared to histogram equalization by local contrast stretching, where several histograms corresponding to each distinct sections of the image are calculated. This information is used to redistribute the lightness values of the image thereby improving the local contrast of an image. This step is performed after the denoising step as the AHE amplifies the noise present in the image.

\section{Level Set Segmentation}

Segmentation being the bottom level processing step in any image analysis and pattern recognition processes, it has to be performed with as much accuracy as possible in order to avoid the propagation of errors throughout the bottom-up processes. The classification of the celestial objects calls for a primary segmentation step that accurately segments the celestial objects $[1,2,5]$. The main constraint for astronomical image segmentation is the lack of well defined and sharp edges. Hence, the segmentation method used should be efficient enough so that, the shape and texture of the blur-edged objects (e.g. Comets, galaxies etc) can be segmented out [17-20].

In the proposed algorithm, the method used for the segmentation of celestial objects is a region based active contour model using level sets. The region based models that uses statistical information is more superior to the edge based model, as the Edge Stopping Function (ESF) fails to lock on to the boundary properly due to the lack of sharp edges. The proposed algorithm uses a region based model following [22] and references therein. This method applies a Signed Pressure Force (SPF) [17] to stop the evolution of the contour, instead of the conventional ESF [21-23].

In this method level set function $\psi$ is initialized to constants rather than the concept of taking level set as a Signed Distance Function (SDF). The values of the level set function remain the same both inside and outside the curve or interface, but the signs are opposite. The SPF function [17] take values in the range [-1,1] based on which the contour expands when inside the object and shrinks when outside the object [22].

Let, $C(q):[0,1] \rightarrow \mathrm{R}^{2}$ be a parameterized planar curve in $X$ where $X$ is a subset of $\mathrm{R}^{2}$ and $I$ be the given image. SPF function is defines as: 


$$
\operatorname{spf}(I(x))=\frac{I(x)-\frac{c_{1}+c_{2}}{2}}{\max \left(\left|I(x)-\frac{c_{1}+c_{2}}{2}\right|\right)}, x \in X
$$

where $c_{1}$ and $c_{2}$ are the average intensities inside and outside the contour, respectively. Mathematical representation for $c_{1}$ and $c_{2}$ are:

$$
\begin{gathered}
c_{1}(\psi)=\frac{\int_{X} I(x) \cdot H(\psi) d x}{\int_{X} H(\psi) d x} \\
c_{2}(\psi)=\frac{\int_{X} I(x) \cdot(1-H(\psi)) d x}{\int_{X}(1-H(\psi)) d x}
\end{gathered}
$$

where the regularized Heaviside function [22] is represented as:

$$
H_{\varepsilon}(z)=\frac{1}{2}\left(1+\frac{2}{\pi} \arctan \left(\frac{z}{\varepsilon}\right)\right)
$$

The level set formulation for an edge based model is:

$$
\frac{\partial \psi}{\partial t}=g|\nabla \psi|\left(\operatorname{div}\left(\frac{\nabla \psi}{|\nabla \psi|}\right)+\alpha\right)+\nabla g . \nabla \psi
$$

where $\alpha$ is the velocity term and $g|\nabla \psi|$ is the ESF.

On Substituting the SPF instead of the ESF in equation (11), the level set formulation becomes (as mentioned in [22]):

$$
\frac{\partial \psi}{\partial t}=\operatorname{spf}(I(x)) \cdot\left(\operatorname{div}\left(\frac{\nabla \psi}{|\nabla \psi|}\right)+\alpha\right)|\nabla \psi|+\nabla \operatorname{spf}(I(x)) . \nabla \psi, x \in X
$$

The term $\operatorname{div}(\nabla \psi /|\nabla \psi|)|\nabla \psi|$ is the curvature term and is used to regularize the level set function $\psi$. For $\psi$ being a level set function, it satisfies the condition $|\nabla \psi|=1$. So the curvature term can be written as $\Delta \psi$ which is the Laplacian of level set function $\psi$. The evolution of a level set function with its Laplacian is equivalent to the Gaussian kernel filtering of the level set on every iteration $[18,19,22]$. The level of regularization can be controlled by the standard deviation of the Gaussian filter. As Gaussian filter is used to regularize the level set, an additional regularization term $\operatorname{div}(\nabla \psi /|\nabla \psi|)|\nabla \psi|$ can be neglected. Moreover, the term $\nabla \operatorname{spf}(I(x)) . \nabla \psi$ in (12) can be removed, as the model uses statistical information of the regions. Hence, the level formulation reduces to:

$$
\frac{\partial \psi}{\partial t}=\operatorname{spf}(I(x)) \cdot \alpha|\nabla \psi|, x \in X
$$

The procedures of the segmentation technique used in this paper are:

a. Initialization of the level set function $\psi$ :

$$
\psi(x, t=0)= \begin{cases}-\rho & x \in \Omega_{0}-\partial \Omega_{0} \\ 0 & x \in \partial \Omega_{0} \\ \rho & x \in \Omega-\Omega_{0}\end{cases}
$$

where $\rho>0$ is a constant, $\Omega_{0}$ is a subset in the image domain $\Omega$ and $\partial \Omega_{0}$ is the boundary of $\Omega_{0}$. 
b. Compute $c_{1}(\psi)$ and $c_{2}(\psi)$ using (9) and (10) respectively.

c. Evolve the level set function based on (13).

d. Gaussian filter regularization of the level set; $\psi=\psi^{*} G_{\sigma}$

e. Check for the convergence of level set evolution. If not, return to step $b$.

This method offers global segmentation which is desirable for the segmentation of astronomical images, as an image frame may contain more than one celestial object. This method is robust to noise and can segment objects with weak edges. Also, the initial contour can be set anywhere in the image. For celestial objects with non homogenous intensities in the interior, this method proves to be efficient over the $\mathrm{C}-\mathrm{V}$ model.

\section{Proposed Algorithm}

Flow graph of the proposed algorithm for the segmentation of the celestial objects from an astronomical image is given below:

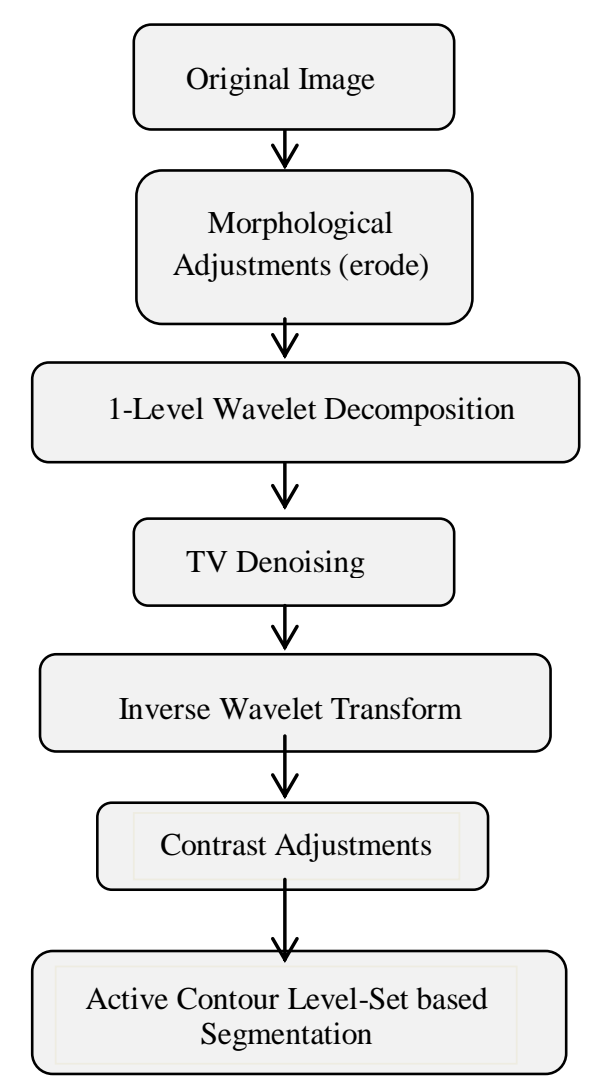

Figure 2: Flow graph of the proposed algorithm

\section{Implementation}

8.1. Image of a galaxy [24] is used to demonstrate the processing steps. The image is converted to a grayscale image for easy processing. The size of the image is taken to be $250 \times 250$. The grayscale image of the galaxy is shown in Fig. 3.

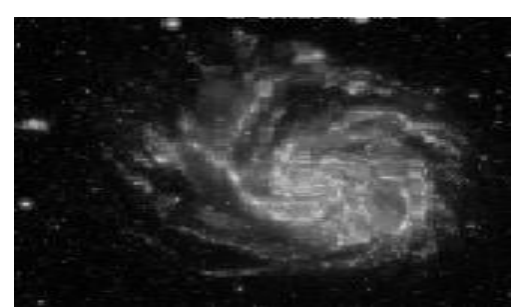

Figure 3: Original image 
8.2. The image has innumerous bright point sources which affects the proper evolution of the segmentation curve. To remove the point sources, a simple morphological processing - erosion, is performed. The structuring element size can be suitably selected by the user, depending on the average size of the point sources. Fig. 4 shows the eroded image using 'disk' of size 2 as the structuring element. This facilitates proper segmentation at the final step. The output of the erosion process is:

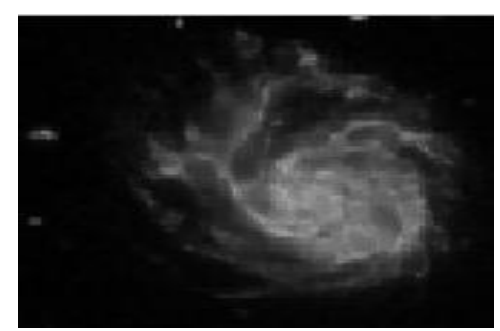

Figure 4: Eroded image

8.3. Wavelet decomposition using Stationary Wavelet Transform (SWT) is performed before the denoising to retain the high frequency edge information to an extent and to avoid translation variance. The wavelet used is Biorthogonal wavelet-'bior2.4'. Fig. 5 shows the approximation and detail coefficient representation of the image.

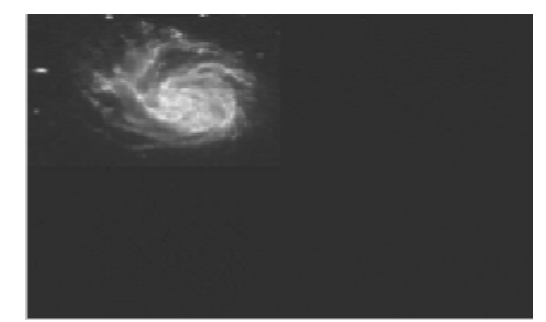

Figure 5: Wavelet decomposition

8.4. TV denoising is performed on each of the decomposed planes i.e., approximation and the detail coefficient planes. Denoising for an iteration of 10 with the control parameter $\lambda=0.1$ is performed. The parameters can be adjusted for various levels of noisiness. A PSNR of 48.69 is obtained for this particular image. Fig. 6 represents the denoised image obtained after performing inverse SWT.

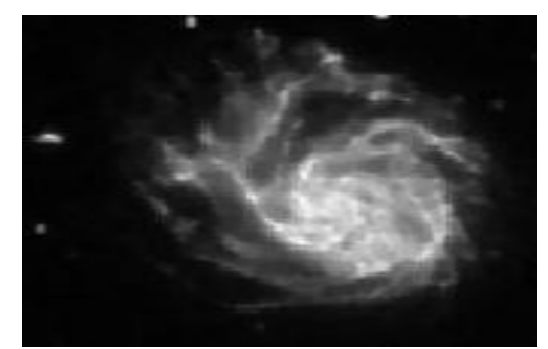

Figure 6: Denoised image after ISWT

8.5. The output of the adaptive histogram equalization applied to the denoised image is given in Fig. 7

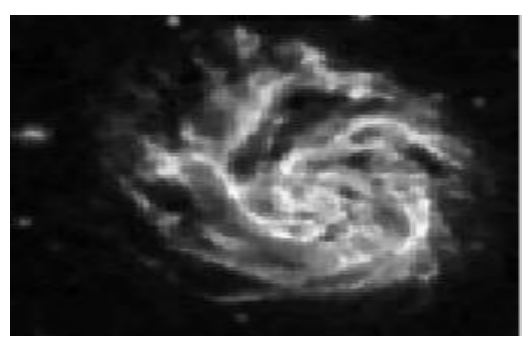

Figure 7: Contrast stretched image 
8.6. The active contour based level set method is applied to the contrast stretched image. The initial contour defined over the image is as shown below:

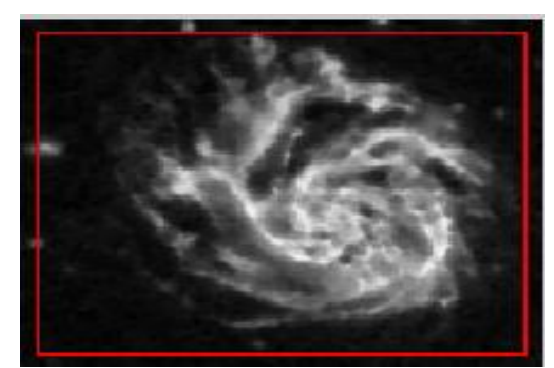

Figure 8: Initialization of level set

The values for the parameters used in the segmentation process are: Standard deviation for the Gaussian filter: $\sigma=1$, number of iteration for the curve evolution $=100$, delta $=10$ and the tuning factor $\mu=.25$. $\mu$ can be varied for properly segmenting different images. The output at the $100^{\text {th }}$ iteration is:

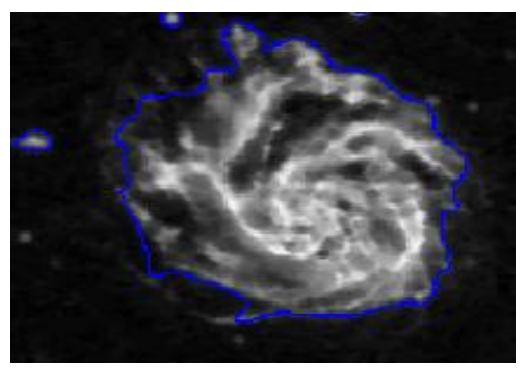

Figure 9:Final contour

The segmented celestial object(s) is extracted from Fig. 9 by setting the background to black.

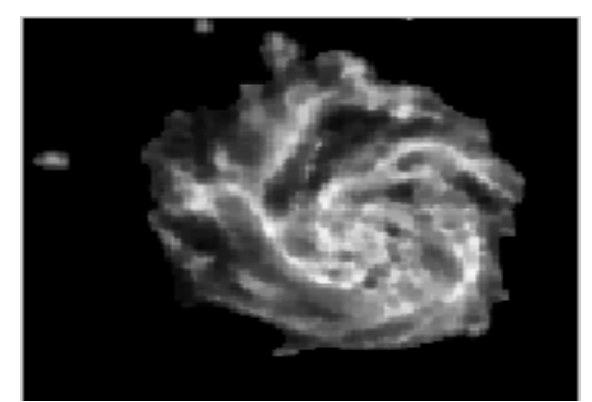

Figure 10:Segmented galaxy

8.7. This image is then projected on to the original image, to retrieve the information content lost during the erode operation. This step regains the original information to an extent. The final output of the segmentation process is shown in Fig.11.

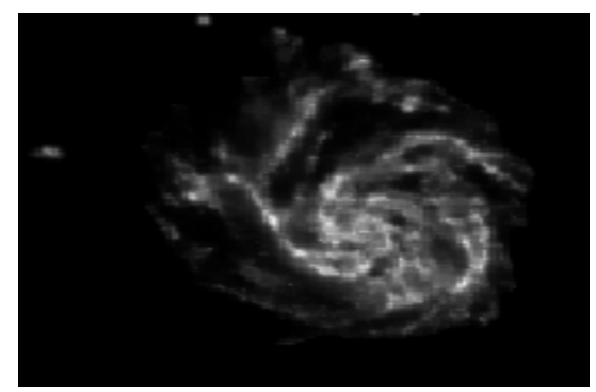

Figure 11:Final segmented image 


\section{Results}

The algorithm is applied to celestial objects like comets, galaxies, asteroids and planets. The processing steps are same as described in Section VII. The output of the proposed algorithm for various celestial objects are:
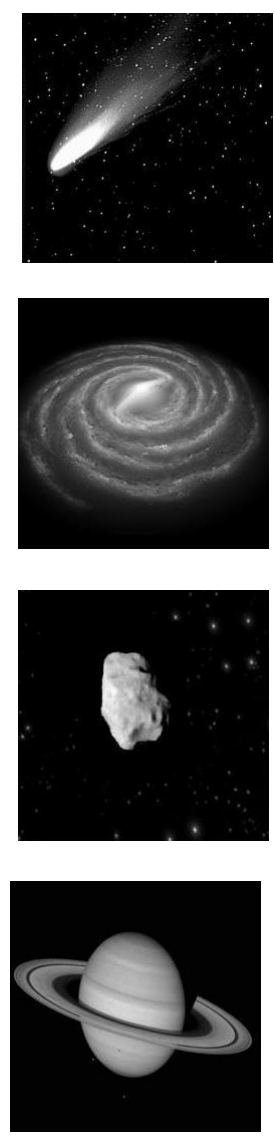

Original image
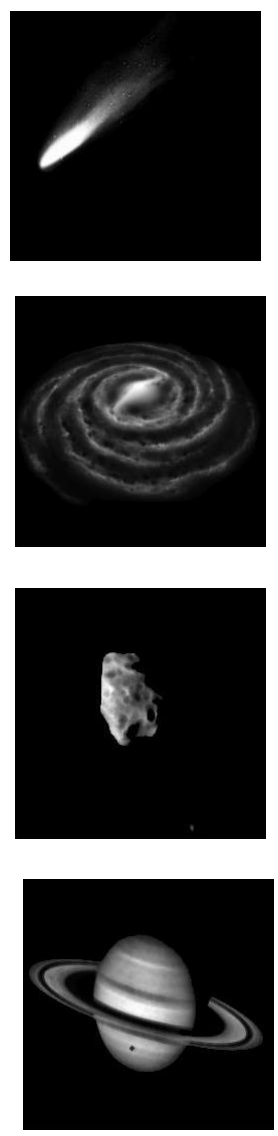

Segmented Output

The subjective analysis of the above results clearly supports the efficiency of the proposed algorithm. The algorithm gives satisfactory results for even the blur- edged objects. The output of this segmentation process can be given as the input for the higher processing steps in Data Mining tasks. The classification of the above segmented astronomical objects can be easily performed with the shape and intensity as the features. The proposed algorithm finds application in the classification and cataloging of celestial objects obtained from satellites or telescopes.

\section{References}

[1] Emmanuel Bertin, Mining pixels: The extraction and classification of astronomical sources, Mining the Sky Eso astrophysics symposia 2001, pp 353-371.

[2] P. Suetnes, P.Fua and A. J. Hanson, Computational strategies for object recognition, ACM Computing Surveys, Vol. 24, pp. 05$61,1992$.

[3] Venkatadri.M, Dr. Lokanatha C. Reddy, A Review on Data mining from Past to the Future, International Journal of Computer Applications, Volume 15-No.7, February 2011, 112-116.

[4] Jean-Luc Starck and Fionn Murtagh, Handbook of Astronomical image and Data Analysis, Springer-Verlag-2002.

[5] E. Aptoula, S. Lef evre and C. Collet, Mathematical morphology applied to the segmentation and classification of galaxies in multispectral images, European Signal Processing Conference (EUSIPCO), Italy, 2006.

[6] Dibyendu Ghoshal, Pinaki Pratim Acharjya, A Modified Watershed Algorithm for Stellar Image, International Journal of Computer Applications, Volume 15-No.7, February 2011, 112-116.

[7] M. Frucci and G. Longo, Watershed transform and the segmentation of astronomical images, In Proceedings of Astronomical Data Analysis III, Naples, Italy, April 2004.

[8] Gonzales, Digital image processing (Pearson Education India, 2009).

[9] K.P Soman, K.I Ramachandran, N.G Reshmi, Insight into wavelet transform, PHI Learning Pvt. Ltd., 2010. 
[10] A. Manjunath, H.M.Ravikumar, Comparison of Discrete Wavelet Transform (DWT), Lifting Wavelet Transform (LWT) Stationary Wavelet Transform (SWT) and S-Transform in Power Quality Analysis, European Journal of Scientific Research, Vol.39, No.4 (2010), pp.569-576.

[11] Xiadong Zhang, Deren Li, Ã Trous wavelet decomposition applied to image edge detection, Geographic information sciences, Vol. 7, No. 2, 2001.

[12] Marta Peracaula, Arnau Oliver, Albert Torrent, Xavier Llado, Jordi Freixenet and Joan Mart, Segmenting extended structures in radio astronomical images by filtering bright compact sources and using wavelets decomposition, 18th IEEE International Conference on Image Processing, 2011.

[13] Vicent Caselles, Total variation based image denoising and restoration, International Congress of Mathematicians, Spain-2006

[14] K.P Soman, R. Ramanathan, Digital signal and image processing-The sparse way, Elsevier India-2012

[15] R.A Hummel, Image enhancement by histogram transformation, Computer vision, graphics and image processing,1977,184-195

[16] Stephen M Pizer, E Philip Amburn, John D Austin, Robert Chromartie et al., Adaptive histogram equalization and its variations, Computer vision, graphics and image processing ,1988.

[17] C.Y. Xu, A. Yezzi Jr., J.L. Prince, On the relationship between parametric and geometric active contours, Processing of $34^{\text {th }}$ Asilomar Conference on Signals Systems and Computers, 2000, pp. 483-489.

[18] Shi, W.C. Karl, Real-time tracking using level sets, IEEE Conference onComputer Vision and Pattern Recognition 2 (2005) 34-41.

[19] P. Perona, J. Malik, Scale-space and edge detection using anisotropic diffusion, IEEE Transaction on Pattern Analysis and Machine Intelligence 12 (1990)

[20] M. Kass, A. Witkin, D. Terzopoulos, Snakes: active contour models, International Journal of Computer Vision 1 (1988) 321-331

[21] V. Caselles, R. Kimmel, G. Sapiro, Geodesic active contours, International,Journal of Computer Vision 22 (1) (1997) 61-79.

[22] Kaihua Zhan, Lei Zhang,, Huihui Song, Wengang Zhou, Active contours with selective local or global segmentation: A new formulation and level set method, Image and Vision Computing 28 (2010) 668-676.

[23] G.P. Zhu, Sh.Q. Zhang, Q.SH. Zeng, Ch.H. Wang, Boundary-based image segmentation using binary level set method, Optical Engineering 46 (2007).

[24] http://archive.stsci.edu visited on $1^{\text {st }}$ October 2012. 